\title{
ADHERENCIAS PENE-PREPUCIALES Y NIVELES DE TESTOSTERONA CIRCULANTES EN ALPACAS
}

\author{
Pablo Chuna M. ${ }^{1}$, Víctor Leyva V. ${ }^{2}$ y Jorge Franco V. ${ }^{1}$
}

\section{Abstract}

In neonate and juvenile male alpacas the prepuce adheres to the glans penis, making extrusion of the penis impossible. The aim of the present research was to determine the relationship between age of prepuce separation and circulating levels of total testosterone. A total of 98 one and two year old male alpacas from Corpacancha (Junín, Perú) were studied. Serum progesterone levels were determined by radioinmunoassay from blood samples and prepuce separation was determined by manual retraction. Data on live weight, testicular size and age were also recorded. Only $4 \%$ of the one year olds $(n=1 / 22)$ and $49 \%$ of the two year olds $(n=37 / 76)$ were free of adhesions and these animals exhibited higher levels of serum testosterone relative to the animals without prepuce separation.

Key words: alpaca, male sexual maturity, prepuce adherence, testosterone

\section{Resumen}

Con el objeto de relacionar la liberación de adherencias pene prepuciales con niveles de testosterona en Alpacas se estudiaron 98 machos Huacaya de uno a dos años de edad. La liberación de adherencias pene prepuciales fue verificada mediante la retracción manual del prepucio. Las muestras sanguíneas fueron colectadas de la vena yugular. Los niveles de testosterona sérica fueron medidos usando RIA de fase sólida. Un 4\% (1/22) de machos de un año y 49\% (37/76) de dos años no presentaron adherencias. Hubo un efecto significativo de la edad en los niveles de testosterona, peso corporal y volumen testicular. Los valores medios de esas variables en animales sin adherencias pene-prepuciales son altos comparados con aquellos que presentan estas adherencias. El análisis de regresión logística mostró que la testosterona afectaba la probabilidad de encontrar adherencias $(\mathrm{P}=$ $0.0155)$, asimismo se ve afectada por el peso corporal y el volumen testicular aunque sin significancia ( $\mathrm{P}=0.0697, \mathrm{P}=0.0681$, respectivamente). Estos datos muestran que la separación prepucial está asociada con los niveles de testosterona circulantes.

Palabras clave: Alpaca, madurez sexual, adherencia pene-prepucial, testosterona

\section{Introducción}

En alpacas como en otras especies de explotación doméstica la presencia de adherencias pene prepuciales es indicadora

1 Práctica privada.

2 Laboratorio de Reproducción - FMV - UNMSM. E.Mail:d170054@unmsm.edu.pe de inmadurez sexual. La liberación de estas estructuras que impiden un libre desplazamiento del pene en el prepucio es un proceso gradual que ocurre con la edad. En efecto, bajo las condiciones de crianza extensiva en los andes, los machos son considerados áptos para iniciar la reproducción a la edad de tres años; esto se debe entre otras razones a que, a ésta edad los machos han completado la liberación de las adherencias pene- 
prepuciales. Sin embargo, hay machos individuales que logran la separación pene prepucial a los dos años y aún más precozmente a un año de edad y que tiene relación con el desarrollo corporal, como es conocido con otras especies como el bovino y ovino. Desde que el proceso bajo consideración debe guardar relación con cambios en la función testicular es de interés averiguar la relación que existe entre niveles séricos de testosterona y la separación pene prepucial; de allí el interés de este estudio.

\section{Matoriales y Métodus}

Las actividades de campo se llevaron a cabo en la SAIS Pachacútec Ltda. N ${ }^{\circ} 7$, ubicada en la localidad de Corpacancha (Junín), situada a $4300 \mathrm{msnm}$, durante los meses de enero a marzo de 1994.

Las determinaciones hormonales se realizaron en el Instituto de Investigaciones de Altura (I.I.A.) de la Universidad Peruana Cayetano Heredia en la ciudad de Lima.

Se muestrearon al azar un total de 22 alpacas machos de la raza «Huacaya» de un año de edad y 76 de dos años de edad. Los animales permanecieron en su hábitat natural alimentándose enteramente de pastos nativos. Solamente, durante la toma de muestras fueron colocados en corrales.

La evaluación de las adherencias pene-prepuciales se realizó a través de la retracción manual del prepucio, sujetando el pene y deslizando hacia atrás la vaina para hacer visible el prepucio.

El grado de adherencia se clasificó según los lineamientos de Sumar (1991), pero tomando en consideración sólo 2 tipos:

Tipo 0: completa adherencia pene-prepucial

Tipo 1: completa liberación de la adherencia pene-prepucial.
Para la medición de testosterona se tomo $5 \mathrm{ml}$. de sangre a cada animal por punción de la vena yugular. Las muestras fueron centrifugadas a 3500 r.p.m. durante 5 minutos para obtener el suero, el cual fue conservado en congelación a $-10^{\circ} \mathrm{C}$ para su posterior determinación hormonal.

Los niveles de testosterona sérica total se determinaron por radioinmunoensayo (RIA) de fase sólida, utilizando kits comerciales del sistema DPC (Diagnostic Product Corporation, CA, Los Angeles). La sensibilidad del ensayo fue de $0.01 \mathrm{ng} / \mathrm{ml}$.

El peso corporal fue registrado con una balanza tipo reloj de $200 \mathrm{Kg}$, con una sensibilidad mínima de $10 \mathrm{~g}$.

Se estimo el peso corporal así como el volumen testicular. Para esto último se consideró largo, ancho y espesor de los testículos. Las medidas fueron tomadas con un vernier calibrado de $0.05 \mathrm{~mm}$ de precisión. El promedio de las medidas de cada par de testículos fue usado para el análisis estadístico.

Los datos de testosterona, peso corporal y volumen testicular de los machos de uno y dos años de edad fueron analizados mediante ANOVA y la comparación de promedios mediante la prueba de Duncan (Steel y Torrie, 1992).

Para examinar la relación entre adherencias con las variables (edad, peso corporal, nivel de testosterona y volumen testicular) se realizó un análisis de regresión mediante el programa estadístico SPSS.

\section{Resullados}

Los resultados sobre niveles de testosterona, peso corporal y volumen testicular se muestran en el Cuadro 1. Nótese que los valores en tuis de un año fueron significativamente menores $(\mathrm{P}<.05)$ que en tuis de dos años. 
Cuadro 1. Valores promedios de los niveles de testosterona sérica (NT), Peso Corporal (PC) y Volumen Testicular (VT) de tuis de uno y dos años de edad.

\begin{tabular}{cccc}
\hline Variables & Tuis 1 año (n) & Tuis 2 años (n) & $\begin{array}{c}\text { Significacia } \\
\text { Estadística }\end{array}$ \\
\hline
\end{tabular}

$\begin{array}{lccc}\text { NT }(\mathrm{ng} / \mathrm{ml}) & 0.8227 \pm 0.2054(22) & 1.4157 \pm 0.1717(76) & * \\ \text { PC }(\mathrm{Kg}) & 35.3864 \pm 1.5797(22) & 47.3487 \pm 0.5620(76) & * \\ \text { VT }\left(\mathrm{cm}^{3}\right) & 6.7809 \pm 1.0232(22) & 17.9503 \pm 0.7952(76) & *\end{array}$

(n) : Número entre paréntesis indican el tamaño de la muestra.

* : $\mathrm{P}<0.05$

Se encontró una gran variación en el porcentaje de liberación con relación a la edad. En los tuis de 1 año sólo el $4 \%$ presentó libre el pene del prepucio, en cambio en los tuis de 2 años un $49 \%$ de las alpacas no presentaron adherencias.

En el Cuadro 2 se observa que los machos sin adherencias pene-prepuciales muestran resultados significativamente $(\mathrm{P}<0.05)$ mayores en niveles de testosterona, peso corporal y volumen testicular que los machos con presencia de adherencias.

Se fijó el siguiente modelo estadístico empleando una regresión logística:

$$
\begin{aligned}
\mathrm{Y}= & 15.9710+0.3360 \text { edad }-0.2746 \text { peso }- \\
& 0.6970 \text { voltest. }-0.9039 \text { test }+0.0128 \\
& \text { peso.voltest. }
\end{aligned}
$$

\begin{tabular}{|c|c|c|c|}
\hline \multirow{2}{*}{ Variables } & \multicolumn{2}{|c|}{ Adherencias pene-prepuciales } & \multirow{2}{*}{$\mathrm{P}<0.05$} \\
\hline & Con adherencias & Sin adherencias & \\
\hline N.T. $(\mathrm{mg} / \mathrm{dl})$ & $0.9616 \pm 0.1088(57)$ & $1.7288 \pm 0.1337(41)$ & ** \\
\hline P.C. $(\mathrm{Kg})$ & $41.9123 \pm 1.0306(57)$ & $48.4878 \pm 0.7702(41)$ & ** \\
\hline V.T. $\left(\mathrm{cm}^{3}\right)$ & $12.2212 \pm 0.9862(57)$ & $19.9217 \pm 1.0176(41)$ & $* *$ \\
\hline
\end{tabular}

Cuadro 2. Comparación de medias de los valores de nivel de testosterona, peso corporal : volumen testicular entre animales con y sin adherencias pene-prepuciales.

En la Cuadro 3, el resultado de ANOVA de la regresión demostró que la testosterona afectaba la probabilidad de encontrar adherencia $(P=0.0153)$. Asimismo se encontró una fuerte tendencia, aunque sin significancia en peso corporal $(\mathrm{P}=0.0697)$ y volumen testicular $(\mathrm{P}=0.0681)$. La prueba de bondad de ajuste del modelo estadístico demuestra que éste es adecuado $(P=0.6103)$.

\section{Discusión}

Nuestros resultados sobre cambios en niveles de testosterona asociados con la edad son similares a los descritos en toros, (Bedair y Thibier, 1979; Lacroix y Pelletier, 1979; Hemeida et al., 1985), carneros (Illius et al., 1976), conejos (Berger et al., 1976), cuyes (Regaudiere et al., 1976) y ratas (Lee et al., 
Cuadro 3. Tabla de análisis de varianza de la regresión logística.

\begin{tabular}{lccccc}
\hline \multicolumn{1}{c}{ Variables } & B & $\begin{array}{c}\text { Error } \\
\text { estandar }\end{array}$ & Wald & $\begin{array}{c}\text { Grados de } \\
\text { libertad }\end{array}$ & Sy \\
\hline Edad & 0.3360 & 0.5199 & 0.4176 & 1 & 0.5181 \\
Peso corporal & -0.2746 & 0.1514 & 3.2906 & 1 & 0.0697 \\
Volumen Testicular & -06970 & 0.3820 & 3.3291 & 1 & 0.0681 \\
Nivel Testosterona & -0.9039 & 0.3726 & 5.8853 & 1 & 0.0153 \\
Peso vs Vol. Test. & 0.0128 & 0.0079 & 2.6190 & 1 & 0.1056 \\
Constante & 15.9710 & 7.0080 & 5.1936 & 1 & 0.0227 \\
\hline
\end{tabular}

1977) y concuerdan con el enunciado de Hafez (1989) en el sentido que el grado de secreción de testosterona aumenta a medida que avanza la pubertad hasta alcanzar un valor constante promedio elevado. En alpacas niveles de testosterona en tuis de un año de edad, más altos que los nuestros fueron descritos por Losno y Coyotupa (1981). Estas diferencias probablemente se deban a variaciones en la toma de muestras. Por otro lado, aunque los niveles hormonales dependen de mecanismos fisiológicos, pueden ser afectados por variaciones ambientales, que no pueden ignorarse al comparar resultados de estudios diferentes.

Con respecto a las adherencias pene prepuciales se conoce que la liberación de éstas ocurre a lo largo de un período prolongado que comienza en el momento del nacimiento y continúa hasta la pubertad (Sorensen, 1982). Similarmente a nuestros resultados en llamas, Olarte y Correón, (1989) encontraron que el desprendimiento pene-prepucial es gradual. Por otro lado nuestros porcentajes de liberación de adherencias son también similares a los descritos en alpacas crías ( $\mathrm{Su}$ mar, 1984) y adultos (Sumar, 1991). En cambio porcentajes mayores a los nuestros en tuis de un año fueron informados por FernándezBaca (1970): 8\%, Nuñez (1994): 12.2\%; y en tuis de dos años por Olarte y Melo (1988): $80.76 \%$, Sumar (1991): $70 \%$ y Nuñez (1994): $59.5 \%$. Estas diferencias probablemente se explican por factores nutricionales (i.e. tuis de 1 año de: Nuñez, 1994, pesaron $39.1 \mathrm{Kg}$; los tuis de dos años de Olarte y Melo (1988), pesaron $70 \mathrm{~kg}$; en el presente estudio los pesos fueron: 33.2955 y $47.2394 \mathrm{~kg}$, respectivamente).

Las diferencias significativas $(\mathrm{P}<0.05)$ en los niveles de testosterona entre animales con y sin adherencias, sugieren el rol de testosterona en la liberación de las adherencias. Esto concuerda con resultados obtenidos por Mather (1986) en bovinos y Korenbrot et al. (1977) en ratas, quienes demostraron que la separación del pene del prepucio es influenciada por los andrógenos circulantes.

Nuestros valores de peso corporal son similares a los encontrados en otros estudios para tuis machos de dos años (Sumar, 1991; Núñez, 1994) pero inferiores a los descritos por Olarte y Melo (1982). Estas diferencias probablemente se explican por variaciones nutricionales. En otras especies domésticas se ha demostrado que la pubertad está relacionada más con el peso corporal que con la edad. Los niveles nutricionales modifican la edad de la pubertad adelantándola o retrasándola según la disponibilidad de alimento. La desnutrición restringe tanto el ritmo de crecimiento como el desarrollo de la función endocrina y puede tener efecto sobre el rendimiento reproductivo.

Las diferencias en peso corporal entre machos sin adherencias pene prepuciales con presencia de dichas adherencias 
sugieren un efecto nutricional. Se conoce que la sobrealimentación acelera el inicio de la pubertad, mientras que el efecto opuesto ocurre con una subalimentación (Coulter, 1986; Amann y Schanbacher, 1983; Mc Donald, 1991). La actividad esteroidogénica y espermatogénica son altas en toros mantenidos con dietas altas de energía (Mather, 1986).

Nuestros resultados sobre volumen testicular en tuis de dos años se aproximan a los reportados por Olarte y Melo (1988). En cambio un volumen testicular mayor fue descrito por Pinares et al. (1985) $\left(37 \mathrm{~cm}^{3}\right)$ y Vivanco et al. (1985) $\left(38.6 \mathrm{~cm}^{3}\right)$, ambos realizados al momento de la liberación pene prepucial (edad aproximada de 13 meses). Es probable que variaciones en peso corporal o efectos estacionales expliquen estas diferencias.

En toros, cerdos y carneros la pubertad está asociada con el rápido crecimiento testicular, cambios en el patrón secretorio de $\mathrm{LH}$ y un incremento gradual en testosterona sanguínea y la iniciación de la espermatogénesis (Amann y Schanbacher, 1983). Nuestros resultados sobre volumen testicular y liberación de adherencias pene prepuciales son consistentes que dichos informes. Estos resultados sugieren la influencia de los niveles hormonales de testosterona en la liberación de las adherencias.

\section{Literatura Citads}

1. Amann, R.P. y B.D. Schanbacher. 1983. Phisiology of Male Reproduction. Journal of Animal Science 57 (Suppl. 2): 330-342.

2. Bedair, G. y M. Thibier. 1979. Peripheral plasma androstenedione and testosterone concentrations in bulls before and during puberty. J. Reprod. Fert. 56:7-10.

3. Berger, M., J. Chazaud, Ch. HeanFaucher, M. De Turchkeim, G.
Veyssiere y C. Jean. 1976. Developmental patterns of plasma and testicular testosterone in Rabbits from birth to 90 days of age. Biology of Reproduction 15:561-569.

4. Coulter, G.H. 1986. Puberty and postpuberal development of beef bulls. In: Current Thrapy in Theriogenology. Diagnosis, Treatment and Prevention of Reproductive Diseases in small and large animals. Ed. Morrow. W.M. Saunders Company. Phyladelphia,

5. Fernández-Baca, S. 1970. Fisiología del Macho. En: Estudios sobre la Reproducción en la Alpaca (Lama pacos). Boletín extraordinario IVITA. UNMSM. 4:33-42.

6. Hafez, E.S.E. 1989. Reproducción e Inseminación Artificial en Animales. 2da. Ed. p.649. Interamericana. México.

7. Hemeida, N.A., Y.R. El Baghdady y M.A. El-fadaly. 1985. Serum profiles of androstenedione, testosterone and LH from birth through puberty in buffalo bull calves. J. Reprod. Fert. 74:311-316.

8. Illius, A.N., N.B. Haynes, K. Purvis $y$ C. Lamming. 1976. Plasma concentrations of testosterone in the developing ram in different social environments. J. Reprod. Fert. 48:17-24.

9. Korembrot, C.C., I.T. Huhtaniemi y R.I. Wiener. 1977. Preputial separation as an external sign of pubertal development in the male rat. Biology of Reproduction 17:298-303.

10. Lacroix A. y J. Pelletier. 1979. Shortterm variations in plasma $\mathrm{LH}$ and testosterone in bull calves from birth to 1 year of age. J. Reprod. Fert. 55:8185.

11. Lee, V.W.K., D.M. De Kretser, B. Hundson $y$ C. Wang. 1975. Variations in serum FSH, LH and testosterone levels in male rats from birth to sexual maturity. J. Reprod. Fert. 42:121-126.

12. Losno, W. y J. Coyotupa. 1981. Testosterona sérica en Alpacas prepúberes. Resúmenes de Proyectos 
de Investigación. Tomo II. p.114. UNMSM. Período 1975-1979. LimaPerú.

13. Losno W., J. Coyotupa y R. GuerraGarcía. 1981. Función endocrina testicular en la Alpaca. Resúmenes de Proyectos de Investigación. UNMSM Período 1975-1979. Tomo II Lima p:108.

14. Mather, E.C. 1986. Puberty in the Bull. In: Current Therapy in Theriogenology. Diagnosis, Treatment and Prevention of Reproductive Diseases in small and large animals. p:339-342. Ed. Morrow. W.B. Saunders Company. Phyladelphia.

15. Mc Donald, L.E. 1991. Endocrinología veterinaria y reproducción. 4ta. Ed. p.551 Interamericana. Mc. Graw Hill. México.

16. Nuñez, J.R. 1994. Relación de la espermatogénesis y desprendimiento del as adherencias pene prepuciales de la alpaca «huacaya» (Lama pacos). Tesis para optar el título de Médico Veterinario y Zootecnistas. Univ. Nac. del Altiplano-Puno.

17. Olarte, V. y Correón, O. 1989. Relación entre edad, peso vivo y tamaño del testículo en el desprendimiento del pene-prepucio en Llamas. VI Conv. Inter. Espec. Camélidos Sudamericanos Oruro-Bolivia.

18. Olarte, V. y M. Melo. 1988. Edad, peso vivo y tamaño del testículo en el desprendimiento pene-prepucio en Alpacas. VI Conv. Inter. Espec. Camélidos Sudamericanos Oruro-Bolivia.

19. Pinares, C., W. Vivanco y W. Foote. 1985. Desarrollo Reproductivo de Alpacas machos del nacimiento a los 18 meses de edad. Resúmenes Científicos del VIII APPA.

20. Regaudiere, N., G. Pelardy, A. Robert y P. Delost. 1976. Changes in the concentrations of testosterone and androstenedione in the plasma and testis of the guinea -pig from birth to death. J.Reprod, Fert. 48:291-300.

21. Sorensen, A.H. 1982. Reproducción Animal: Principios y Prácticas. Mc GrawHill S.A. México. 530p.

22. Steel, R.G.D. y J.H. Torrie. 1992. Bioestadística: Principios y Procedimientos. 2da. edición. p.620. Mc Graw Hill S.A. México.

23. Sumar, J. 1984. Fisiología Reproductiva de la Alpaca. Boletín Científico de La Raya (IVITA). UNMSM, 1:36.

24. Sumar, J. 1991. Fisiología de la Reproducción del Macho y Manejo Reproductivo. In: Avances y Perspectivas del Conocimiento de los Camélidos Sudamericanos. Ed. S. Fernández-Baca. FAO. Santiago de Chile,. p:111-143.

25. Vivanco, W., W. Foote y $C$. Pinares. 1985. Estudio del Desarrollo de algunas características reproductivas en alpacas machos huacaya en la Sierra Central del Perú, nacimiento, a los 13 meses de edad y sus correlaciones con el desarrollo corporal. Resúmenes Científicos del VIII APPA.

26. Watson R.H., C.S. Sapsford y I. Mc Cance. 1956. The Development of the testis, epididymis, and penis in the young Merino ram. Australian Journal of Agricultural Science 7: 574-590. 